\title{
1 Airborne point counts: a method for estimating songbird abundance with drones
}

3 Authors: McKenzie D. Somers ${ }^{1}$, Darren B. Glass ${ }^{2}$, Marisa A. Immordino ${ }^{1}$, Precious S. Ozoh ${ }^{1}$,

4 Lauren B. Sherman ${ }^{1}$, and *Andrew M. Wilson ${ }^{1}$,

${ }^{1}$ Environmental Studies Department, Gettysburg College, Pennsylvania 17325, USA

$7 \quad{ }^{2}$ Mathematics Department, Gettysburg College, Pennsylvania, 17325, USA

$9 \quad *$ corresponding author: awilson@gettysburg.edu

\section{Acknowledgements}

12 We thank Alyssa Kaewwilai and Francesca Garrison for help testing fieldwork protocols. Our

13 study site is on the land of the Susquehannock peoples.

14 Funding statement: This work was supported by the Cross-Disciplinary Science Institute at

15 Gettysburg College (X-SIG) and endowed funds from the Cargill Foundation.

16 Ethics statement: The drone was flown with the permission of Gettysburg College, under

17 restrictions placed by the Federal Aviation Authority’s Part 107 Remote Pilot License. The use

18 of State Game Lands 249 was with granted permission of the Pennsylvania Game Commission.

19 Author Contributions: A.M.W. formulated the research question. M.A.I., P.S.O., L.B.S,

20 M.D.S. and A.M.W. developed fieldwork protocols and conducted the fieldwork. D.B.G.

21 developed analytical methods. All authors contributed to writing and editing the manuscript. 


\section{Abstract}

23 Using drones to conduct airborne bioacoustic surveys is a potentially useful new way to estimate

24 the abundance of vocal bird species. Here we show that by using two recording devices

25 suspended from a quadcopter drone it is possible to estimate distances to birds with precision. In

26 an experimental test, the mean error of our estimated distances to a broadcast song across 11

27 points between 0 and $100 \mathrm{~m}$ away was just $3.47 \mathrm{~m}$. In field tests we compared 1-minute airborne

28 counts with 5-minute terrestrial counts at 34 count locations. We found that the airborne counts

29 yielded similar data to the terrestrial point counts for most of the 10 the songbirds included in our

30 analysis, and that the effective detection radii were also similar. However, airborne counts

31 significantly under-detected the Northern Cardinal $\left(\chi_{9}^{2}=22.8\right.$, post-hoc test $\left.P=0.007\right)$, which

32 we attribute to a behavioral response to the drone. Airborne counts work best for species that

33 vocalize close to the ground and have high frequency-range songs. Under those circumstances,

34 airborne bioacoustics could have several advantages over ground-based surveys, including

35 increased precision, increased repeatability, and easier access in difficult terrain. Further, we

36 show that it is possible to do rapid surveys using airborne techniques, which could lead to the

37 development of much more efficient survey protocols than are possible using traditional survey

38 techniques.

40 Keywords: distance sampling, drones, bioacoustics, time difference of arrival, songbird 


\section{Lay Summary}

42 - We show that it is possible to estimate the distance of singing birds from a drone, which

43 then allows bird counts to be converted to true abundance or population densities.

44 - Using drones to count birds allows researchers to survey areas that may be difficult or

45 dangerous to access on foot.

46 - Airborne counts are potentially a highly efficient and highly repeatable way to estimate

$47 \quad$ populations of vocal bird species. 


\section{Introduction}

49 Drones are now widely used in ecology to locate, count, or track organisms, and to map resources (Nowak et al. 2019). Most studies that have used drones to count birds have focused on

51 larger species such as seabirds (Hodgson et al. 2018) and waterbirds (Afán et al. 2018, Pöysä et

52 al. 2018) which are more likely to be detected in aerial imagery. The use of drones for

53 bioacoustic surveys is less well-established, but initial studies have shown promising results for

54 songbirds (Wilson et al. 2017) and bats (Kloepper and Kinniry 2018, Fu et al. 2018, August and

55 Moore 2019). Drone-based bioacoustic studies have the potential to harness the advantages of

56 bioacoustic techniques using automated recording units (ARUs) (Darras et al. 2018), together

57 with increased mobility and ease of access in difficult terrain. Increased access could reduce

58 habitat biases that are prevalent in bird survey data (Betts et al. 2007, Leitão et al. 2011). Other

59 advantages of ARUs include obtaining a permanent record that can be analyzed/reanalyzed at a

60 future date, and reducing observer bias (Campbell and Francis 2013, Shonfield and Bayne 2017).

61 Further, while it is true that drones can cause disturbance to wildlife (Mulero-Pázmány et al.

62 2017), drones also have the potential to reduce disturbance caused by field biologists wandering

63 through sensitive habitats (Christie et al. 2016, Borrelle and Fletcher 2017).

64

65 A previous study found that using inexpensive recording devices suspended from quadcopter

66 drones to count songbirds produced counts that were broadly similar to those obtained by typical

67 ground-based point count protocols (Wilson et al. 2017). However, the aim of many bird survey

68 techniques is to estimate bird abundance, usually standardized to a given number of individuals

69 per unit of area (Bibby et al. 2000), which then allows more direct comparison across species,

70 locations, habitats, or time (Gregory et al. 2004). For airborne bioacoustic techniques to provide 
71 a useful alternative to ground-based survey methods (e.g. point counts, transects, territory

72 mapping), the ability to estimate abundance within a given spatial area is highly desirable.

74 Here, we show that it is possible to estimate radial distances of bird vocalizations from a drone

75 using two small inexpensive recorders, and a prosumer-grade quadcopter. We utilize the time

76 difference of arrival technique (TDoA) (Mennill et al. 2006) to estimate distances to vocalizing

77 birds on a horizontal plane either at ground-level, or at given heights above the ground.

78 Estimation of distances to birds would allow the application of Distance Sampling techniques

79 (Buckland et al. 2005) to estimate population densities. We show an experimental proof of

80 concept and a field application, where we compare density estimates from airborne counts with

81 those of traditional terrestrial point counts and territory/spot-mapping (Bibby et al. 2000).

\section{METHODS}

\section{Equipment}

85 We used a DJI Mavic Pro quadcopter programmed to fly missions autonomously using the Litchi

86 app (VC Technology Ltd.) for iOS on an iPhone SE. We used aftermarket “Low-noise” DJI

87 propellers, which reduce drone noise by $\sim 4 \mathrm{~dB}$ (Valle and Scarton 2019). Our recording devices

88 were lightweight and inexpensive Zoom H1 Handy recorders, which weigh just 95 g, including

89 battery, microSD card and, importantly, a windshield. It should be noted that this model of

90 recorder was also chosen because of its cardioid pickup pattern, which reduces sound pickup

91 from the rear of the microphones, i.e. in the direction of the drone when the microphone is

92 directed at the ground. We attached the recorders to the drone using a system of fishing line, zip

93 ties, and small carabiners. 


\section{Measuring distances}

95 To enable the use of time difference of arrival to estimate distances, the recorders had to be

96 sufficiently far apart that time differences were measurable, and they had to be suspended below

97 the drone to reduce excessive drone noise on the recordings. Previous tests showed that

98 suspending recorders up to $15 \mathrm{~m}$ below the drone was manageable in the field, where great care

99 is required to avoid entangling the fishing line on the drone or vegetation. Our system placed one

100 recorder $7.5 \mathrm{~m}$ below the drone, and the second recorder $15 \mathrm{~m}$ below the drone. To calculate

101 TDoA, the two recorders needed to be time-synchronized. We did this manually by placing the

102 microphones of the two recorders $\sim 2 \mathrm{~cm}$ apart and playing a tree cricket (Oecanthus sp.)

103 recording from an iPhone placed between them, thereby allowing the two recordings to be

104 clipped to the same time point (to <1 mS accuracy) in Audacity (Audacity Team 2019).

105 We then merged the two recordings in Audacity to make a single stereo audio track, which

106 included time-differences in sound sources. TDoAs were measured manually from spectrograms

107 (Hanning window with 512 samples and 89\% overlap) in program Raven Pro 1.5 (Bioacoustics

108 Research Program 2014).

109

110 With TDoAs measured we were then able to apply the Pythagorean Theorem to calculate the

111 radial distance from the drone location to the sound source $(x)$, in meters across the ground, using

112 the following formula:

113

$114 x=\frac{\sqrt{a^{4}+b^{4}+\Delta^{4}-2 a^{2} b^{2}-2 b^{2} \Delta^{2}-2 a^{2} \Delta^{2}}}{2 \Delta} \quad$ [Formula 1] 
115 where $a$ is the altitude of the bottom recorder (in meters), $b$ is the altitude of the top recorder

116 (meters) and $\Delta\left(\Delta_{\mathrm{cd}}\right.$ in Figure 1$)$ is the estimated difference in the Euclidean distance from the

117 recorders to the sound source $(c-d) . \Delta$ is calculated by multiplying the TDoA by the speed of

118 sound at a given air temperature $\left(S O S_{t}\right)$. Hence, for any given TDoA, we could estimate the

119 radial distance $(x)$ between the point under the drone and a sound source.

121 Because we were only able to measure time difference to whole mS (the measurement limit in

122 Raven Pro), we must assume that the actual time difference was in a range of the measured

123 TDoA $\pm 0.5 \mathrm{mS}$. Hence, we calculate the distance of the bird to be in a range between a lower

$124\left(x_{l}\right)$ and upper $\left(x_{u}\right)$ limits, and assuming that birds are randomly distributed within the band

125 between those distances, a single estimate can be derived by estimating the median distance

126 between the two:

$x_{m}=\sqrt{\frac{\left(\pi x_{l}^{2}\right)+\left(\left(\pi x_{u}^{2}-\pi x_{l}^{2}\right) / 2\right)}{\pi}} \quad$ [Formula 2]

$130 x_{m}$ is therefore the estimated radial distance to the bird, which can used in distance sampling, or

131 fixed radius distance abundance estimation from airborne point counts.

133 We used MS Excel to estimate radial distances to the sound source based on the above formulae.

134 A spreadsheet that allows the user to estimate distance from measured TDoAs based on inputted 135 air temperature $(t)$, and recorder heights $(a, b)$ is provided in Appendix 1. It is important to note 136 that Formula 1 assumes that the sound source is on a horizontal plane at ground level. However, 
137 this assumption can be changed, for example, it can be assumed that a certain species typically

138 sings from vegetation $4 \mathrm{~m}$ above ground-level, hence, heights $a$ and $b$ would be reduced by $4 \mathrm{~m}$.

139 Height of bird from the ground is therefore included in the spreadsheet as an additional

140 parameter, that can be varied according to species and habitat. To test how sensitive the

141 technique would be to uncertainty in estimating the height of birds from the ground we assumed

142 an air temperature of $20^{\circ} \mathrm{C}$ and recorder heights at 40 and $47.5 \mathrm{~m}$, and varied the height of the

143 bird from the ground parameter from 0 to $10 \mathrm{~m}$. We also used the spreadsheet to evaluate the

144 effects of using different distances between the two recorders on TDoAs, which might be varied

145 according to the study species and habitats, and research needs.

\section{Experimental test}

148 To test the method we broadcast a bird song recording (American Robin Turdus migratorius;

149 source: Macauley Library. 2014) at $90 \mathrm{~dB}$ at $1 \mathrm{~m}$ (measured using an Extech 407730 sound level

150 meter) from paired Aomais Go speakers, placed on the ground. We then flew a mission where

151 the drone hovered for 1-minute at altitudes of $55 \mathrm{~m}$ at 11 locations in $10 \mathrm{~m}$ increments along a

152 transect, $0 \mathrm{~m}$ to $100 \mathrm{~m}$ across the ground from the broadcast speakers. The experiment was

153 conducted on sports fields at Gettysburg College, PA, where background anthropogenic and

154 biological noise was minimal. The temperature was $21^{\circ} \mathrm{C}$ and winds were light.

156 Field test

157 With proof of concept established in our experiment, we sought to test the method in a field

158 study, where we compared it to two traditional songbird survey techniques: point counts, and

159 territory/spot mapping (Bibby et al. 2000). These survey methods are henceforth referred to as 
"mapping” and "terrestrial counts", and surveys using the drone are referred to as "airborne

161 counts”. The study was conducted in a section of State Game Lands 249, Adams County,

162 Pennsylvania; 140 hectares of grassland and shrubby fields, with some small woodlots and

163 wetlands $\left(39.9374^{\circ} \mathrm{N},-77.1774^{\circ} \mathrm{W}\right)$. Two tracks provide vehicular access to the site, but

164 otherwise, the area has little human disturbance. Recreational drone flying is not permitted at the

165 site. We focused our surveys on ten songbird species known to be present in sufficient numbers

166 (>30 individuals): the Willow Flycatcher (Empidonax traillii), House Wren (Troglodytes aedon),

167 American Robin (Turdus migratorius), Field Sparrow (Spizella pusilla), Song Sparrow

168 (Melospiza melodia), Eastern Towhee (Pipilo erythrophthalmus), Yellow Warbler (Setophaga

169 petechia), Common Yellowthroat (Geothlypis trichas), Northern Cardinal (Cardinalis

170 cardinalis), and Indigo Bunting (Passerina cyanea).

171

172 For the airborne and terrestrial point counts we surveyed 34 pre-determined locations evenly

173 spaced on 200-meter grid (data from a $35^{\text {th }}$ point were dropped due to excessive wind noise on

174 drone recordings). Terrestrial and airborne counts were conducted on the same day, between $31^{\text {st }}$

175 May and $6^{\text {th }}$ June 2019, and between 6:00am and 9:00am. Weather conditions were suitable for

176 both count methods, i.e. no rain, and wind less than a force 4 (15 mph) on the Beaufort scale

177 (NOAA). Terrestrial counts were of 5-minute duration, with observations categorized by minute

178 of first detection. Estimations of distances to birds seen or heard were aided by a laser range

179 finder. Bird detections were categorized as singing, calling, or visual only. Terrestrial counts

180 were all conducted by a single very experienced point count surveyor (A.M.W.). 
182 For airborne counts, we hovered the drone at $55 \mathrm{~m}$ above ground-level for 1-minute. As in the

183 experimental test, the two recorders were suspended $7.5 \mathrm{~m}$ and $15 \mathrm{~m}$ below the drone, hence, the

184 recorders were $40 \mathrm{~m}$ and $47.5 \mathrm{~m}$ above ground-level. The drone was launched from at least 100

185 m away from the count locations. We conducted between two and five adjacent airborne counts

186 in succession, which was readily done on a single battery. Based on personal observation and

187 understanding of the habitat at our site, we estimated that most of the singing birds were in low

188 vegetation. In our distance calculations we estimated that Willow Flycatcher, Field Sparrow,

189 Song Sparrow, and Common Yellowthroat were $2 \mathrm{~m}$ above ground level, House Wren and

190 Yellow Warbler 3 m, Eastern Towhee 4 m, American Robin and Northern Cardinal 5 m, and

191 Indigo Bunting 6 m. In program Raven we labeled each bird on the airborne count recordings

192 with a unique code so that we could track song output and possible movement for each

193 individual, based on song-bout spacing, unique song patterns, apparent volume (from

194 spectrograms) and calculated distances. We found very few instances of ambiguity when it came

195 to identifying individual birds, based on these factors, even for the most abundant species.

197 To provide context for our point count data we conducted a territory mapping study of the entire

198 site, visiting all areas on at least four occasions between mid-May and early July 2019. While the

199 optimal number of visits for territory mapping is 10, a minimum of 4 will suffice (Gregory et al.

200 2004). In order to ensure the entire study area was surveyed as quickly as possible, teams of

201 researchers surveyed different sections of the study area simultaneously. We estimated the

202 number of territories using standard protocols (Bibby et al. 2000), with a lower estimate where

203 territory clusters needed observations on two visits at least 10 days apart, and a higher estimate

204 where single visit detections were also included as territories. 


\section{Analytical methods}

207 We estimated the density of singing birds from the terrestrial and airborne count data using the $\mathrm{R}$

208 package Rdistance (Miller et al. 2019). Half-normal, Hazard Rate and Negative Exponential

209 detection functions were fitted and the best model was selected using AIC. Density estimates

210 were compared with the estimated number of territories to see whether there was broad

211 agreement in abundance estimates among the three bird survey techniques. Calculated Effective

212 Detection Radii for terrestrial and airborne counts indicate whether the airborne counts are able

213 to capture song detections over a similar area to a fieldworker on the ground. We tested to see

214 whether there were differences in the species make-up of detections between airborne counts and

215 5-minute terrestrial counts using chi-square tests and post hoc tests of residuals with Bonferroni

216 adjustment, using $\mathrm{R}$ package chisq.posthoc.test (Ebbert 2019)

218 RESULTS

\section{Experimental results}

220 Our experimental test showed that we were able to estimate the distance to the American Robin

221 song broadcast with a high degree of accuracy; the mean absolute error was $3.47 \mathrm{~m}$, which

222 resulted in a mean overestimate of distances of $1.9 \mathrm{~m}$ (Figure 2).

224 We found that varying the distance between the two recorders affects the precision with which

225 TDoAs can be measured. We note that the TDoA is maximized when the sound is directly below

226 the drone, in which case the TDOA is given by $S^{*}(b-a)$. In particular, if the recorders were 5

$227 \mathrm{~m}$ apart, the maximum feasible TDoA at an air temperature of $20^{\circ} \mathrm{C}$ is $15 \mathrm{mS}$, compared with 22 
228 at $7.5 \mathrm{~m}$, and 29 at $10 \mathrm{~m}$. More generally, one can directly show from the formula that as the

229 distance between recorders is increased the formula for $x$ becomes less sensitive to errors in the

230 measured value of TDoA (range bars in Figure 3A).

231

232 The same computation shows that raising the height of the drone, while maintaining the distance

233 between the two recorders constant, will make the formula less sensitive to error calculations.

234 Uncertainty with respect to the height of birds off the ground will have rather little effect on

235 distance estimation for birds close to the drone, but estimates would be increasingly uncertain at

236 greater distance (Figure 3B). For example, if a TDoA of $10 \mathrm{mS}$ is measured, the estimated

237 distance to a bird on the ground is $85.3 \mathrm{~m}$, compared with $75.6 \mathrm{~m}$ if it is assumed the bird is $5 \mathrm{~m}$

238 off the ground, and $65.8 \mathrm{~m}$ if the bird is $10 \mathrm{~m}$ off the ground.

240 Field test

241 We detected 603 song bouts across our 10 target species on the 34 airborne counts; of which 369

242 song bouts were estimated to be by birds within a 100 m radius of the point location (Table 1).

243 Detections beyond $100 \mathrm{~m}$ were discarded from further analysis due to the possibility of

244 duplication between adjacent points. We found that song output was consistent throughout the

245 one-minute airborne counts, with no indication of a curtailment of song activity in the drone’s

246 presence (Figure 4A). We attributed the 369 song bouts to 120 different individuals (Table 1), of

247 which 73\% were detected within the first 20 seconds (Figure 4B).

249 The overall patterns of detections from airborne counts were similar to those from terrestrial

250 counts (Figure 5); there was evidence of declining detection rates at distances of greater than 60 
251 m on both point count surveys. Total song detection were higher on airborne counts than in the

252 first minute of terrestrial counts, but lower than the number of individuals detected in 5 minutes

253 (Table 1). Distance sampling derived density estimates for the two most numerous species-

254 Field Sparrow and Song Sparrow_-were higher on airborne counts than during the first minute

255 of terrestrial counts, and very comparable with those of five-minute point counts (Figure 6).

256 Density estimates of singing males were lower for both point count methods than the estimated

257 densities of territories derived from mapping (Figure 6). The effective detection radii for Field

258 and Song Sparrows were similar for the airborne counts and terrestrial counts (Table 2).

260 For six of the 10 species the 1-minute airborne counts picked up more singing birds than the first

261 minute of the terrestrial count, and for three species, the airborne counts were higher than after

262 the full 5-minutes of terrestrial counts (Table 1; Figure 5). There was a significant difference in

263 the species composition of detections from airborne counts compared to 5-minute terrestrial

264 counts $\left(\chi_{9}^{2}=22.8, P=0.007\right)$, with relatively more Field Sparrows (post hoc tests, $P=0.045$ ),

265 and fewer Northern Cardinals (post hoc tests, $P=0.016$ ) on airborne counts. 


\section{DISCUSSION}

269 Our initial tests prove the concept that it is possible to estimate distances to vocalizing birds with

270 a pair of synchronized recorders suspended from a drone. Our estimates of error are very modest

271 compared to the average observer error of $19 \mathrm{~m}$ estimated in a field study by (Alldredge et al.

272 2007), but we acknowledge that our experimental test was under controlled conditions.

274 Our field test showed that airborne counts were able to produce data suitable for density

275 estimation that was similar, overall, to that obtained by an experienced fieldworker conducting

276 ground-based point counts. It is important to note that some species appear to have been under-

277 detected on airborne counts, notably the Northern Cardinal. Although our data did not show a

278 decline in song output during the 1-minute drone flights, it is possible that some individual birds

279 stopped singing as the drone approached. Interestingly, in a previous study of seven song bird

280 species, the Northern Cardinal was found to be the most sensitive to drone noise (Wilson et al.

281 2020). However, as drone technology has matured, small quadcopters have become steadily

282 quieter, and smaller, and further technical developments (e.g. Hioka et al. 2019) could result in

283 drones that are quiet enough to greatly reduce, if not eliminate, noise disturbance effects.

285 That our density estimates from point counts were very different to those derived from territory

287 protocols, have been widely reported (Shankar Raman 2003, Howell et al. 2004, Newell et al.

288 2013). The advantages of point counts (either terrestrial or airborne) over mapping is that they

289 don't require access to an entire study area (Gregory et al. 2004), and they are generally much

290 more time-efficient. Even though our mapping study was scaled back to just four visits to each 
291 part of the study area, it required 66 hours of fieldwork, and many hours of collating and

292 analyzing maps.

293

294 There are several potential sources of error in our airborne count distance estimates. One of the

295 main sources of error is whether the altitude of the recorders is accurate, and how high off the

296 ground the vocalizing bird is perched (i.e. accuracy of distances $a$ and $b$ ). Drone GPS accuracy is

297 now exceptional, with the manufacturer of the DJI Mavic claiming vertical accuracy of $0.1 \mathrm{~m}$

298 (https://www.dji.com/mavic/info), so we assume that error due to GPS accuracy is trivial.

299 However, the fact that the height of a bird from the ground is not known could introduce

300 significant imprecision and bias into distance estimates. Our method allows estimated perching

301 heights of a particular species from the ground to be incorporated into calculations. Heights

302 could be measured by field validation or estimated using expert opinion. Even so, we currently

303 recommend that our technique is only valid for birds that reliably vocalize from the ground or in

304 low vegetation, and hence is potentially useful for birds of open habitats, including grasslands,

305 wetlands, bare ground, and shrub/scrub. We caution that over-estimating the perching height of

306 birds would lead to an under-estimate of bird densities, because it would be assumed that the bird

307 was further away than it actually was (see Figure 3B).

308

309 Another assumption of our technique is that birds vocalize from a horizontal plane. Uneven

310 topography and slopes would therefore introduce inaccuracy into distance estimates, the extent

311 of which merit further investigation. We note that in the case of even and modest slopes, upslope

312 and downslope errors should balance, hence distance estimates will be less precise, but not 
necessarily biased. Applying our technique in topographically complex locations, where errors

314 could include significant biases, would be more challenging.

316 A more general limitation of airborne bioacoustics is that drone noise on recordings may mask

317 low frequency songs and calls (Wilson et al. 2017). For such species only those closest to the

318 drone are likely to be detected, reducing the effective sampling distance of this survey method,

319 which could reduce sample sizes. There are, of course, other complications inherent with using

320 drones for survey work including ethics safety, the requirement for additional training and

321 possibly licensing for drone pilots, and the need for relatively calm weather conditions (Linchant

322 et al. 2015, Wallace et al. 2018). However, we have not found any of these to inhibit our ability

323 to use these techniques in Pennsylvania, USA.

325 As with more general use of ARUs, airborne bioacoustics has some clear disadvantages when 326 compared with traditional bird counts techniques. The most important is that recorders do not 327 pick up visual detections, and hence will undercount species that rarely vocalize when compared 328 with counts by a field ornithologist. In some instances, it may be difficult to be certain how many 329 individual birds of each species are audible in a recording, but airborne count using our method 330 have an advantage over ARUs - the ability to estimate distances provides an additional clue 331 when the analyst is trying to decipher how many birds are detected on a recording.

\section{Future developments}

334 Importantly, our airborne counts were of just one minute duration, showing that rapid density

335 estimation may be possible using drones. Further, as 74\% of singing bird detections were within 
the first 20 seconds, we believe that it may be possible to use our method to conduct a rapid

337 series of airborne counts in a very short period of time. For example, it would be feasible to fly

338 two adjacent drone missions, each with up to 15 x 30-second counts spaced every $200 \mathrm{~m}$, for a

339 total of 30 point counts densely covering an area of $1.2 \mathrm{~km}^{2}$ in point counts in just one hour of

340 field work. This highly efficient data gathering makes it more feasible to repeat point counts over

341 a day or season, which potentially allows for more robust population estimates using occupancy

342 models (Royle and Nichols 2003, Hayes and Monfils 2015) or spatially replicated N-mixture

343 models (Royle 2004). An added advantage of using drones for aerial bioacoustics surveys under

344 those circumstances is that they are highly replicable, both in terms of location and duration (due

345 to the ability to precisely program missions), and because audio recordings provide a permanent

346 record of bird song that can be analyzed by multiple observers after the fact (Shonfield and

347 Bayne 2017).

349 Although our analysis shows that drones could be a very efficient way to collect data on songbird abundance, it is important to consider the extra analytical time that the method would entail,

351 when compared to terrestrial counts. It took more than one hour of data analysis per airborne

352 count to identify each song bout and then estimate the distance to the bird using the TDoA

353 method - but note that we did localize every song bout, whereas it may be only necessary to

354 localize the first detection.

356 In addition to trying very rapid assessment with short duration point counts, our technique could 357 easily be modified to fly airborne line transects, which would be even more time-efficient. Two 358 potential pitfalls of that approach are that the recorders may not suspend vertically from a 
359 travelling vehicle, and drone noise increase with speed - even a slow-moving quadcopter is

360 noticeably noisier than one hovering.

361

362 Recent advances have shown that the distances of vocalizing birds from automated recording

363 units can be estimated from relative sound levels (Yip et al. 2019), which could also be measured

364 using airborne bioacoustics. Combining TDoA based distance estimates with those derived from

365 measurements of sound levels offers an intriguing avenue for technique validation, and for

366 potentially developing a robust technique that combines estimates from the two techniques to

367 reduce uncertainty.

369 This initial study used off-the-shelf and inexpensive prosumer-level drones and recorders.

370 Results might be greatly improved with custom-designed drones, to reduce drone noise, and

371 custom-built audio recorders. A recorder with the ability to simultaneously record two tracks

372 from input lines of different lengths, would negate the need for manually synchronizing the

373 tracks from the two recorders. We were not able to find a lightweight recording device that

374 satisfied those requirements, but there are options that would be suitable for larger drones that

375 are capable of carrying more payload. However, larger drones are noisier, so the trade-off

376 between payload and the potential for noise disruption needs careful consideration. 


\section{Conclusions}

379 We show that it is feasible to estimate distances of vocalizing birds from a drone, which

380 therefore allows robust estimation of abundance in the same way that traditional bird surveys do.

381 This technique could be applied to places that are difficult or dangerous for point count

382 technicians to access. Further, our study shows that gathering bird abundance data this way could

383 be highly efficient, so this technique may be of broader interest. While the technique outlined

384 here is subject to various assumptions, and will likely not work for all species or habitats, we

385 conclude that with further development of equipment and analytical methods, airborne

386 bioacoustics could provide a useful new way to estimate the abundance of vocal bird species. 


\section{Literature Cited}

389

390

391

392

393

394

395

396

397

398

399

400

401

402

403

404

405

406

407

408

409

410

Afán, I., M. Máñez, and R. Díaz-Delgado (2018). Drone Monitoring of Breeding Waterbird Populations: The Case of the Glossy Ibis. Drones 2:42.

Alldredge, M. W., T. R. Simons, and K. H. Pollock (2007). A Field Evaluation of Distance Measurement Error in Auditory Avian Point Count Surveys. Journal of Wildlife Management 71:2759-2766.

August, T., and T. Moore (2019). Autonomous drones are a viable tool for acoustic bat surveys. bioRxiv:673772.

Betts, M. G., D. Mitchell, D. A. W., and J. Bêty (2007). Uneven Rates of Landscape Change as a Source of Bias in Roadside Wildlife Surveys. Journal of Wildlife Management 71:2266.

Bibby, C. J. (Colin J. ., Ecoscope Applied Ecologists., British Trust for Ornithology., Royal Society for the Protection of Birds., and BirdLife International. (2000). Bird census techniques. Academic Press.

Bioacoustics Research Program (2014). Raven Pro: Interactive Sound Analysis Software. The Cornell Lab of Ornithology, Ithaca, New York.

Borrelle, S. B., and A. T. Fletcher (2017). Will drones reduce investigator disturbance to surfacenesting birds? Marine Ornithology 45:89-94.

Buckland, S. T., D. R. Anderson, K. P. Burnham, J. L. Laake, S. T. Buckland, D. R. Anderson, K. P. Burnham, and J. L. Laake (2005). Distance Sampling. In Encyclopedia of Biostatistics. John Wiley \& Sons, Ltd, Chichester, UK.

Campbell, M., and C. M. Francis (2013). Using Stereo-Microphones to Evaluate Observer Variation in North American Breeding Bird Survey Point Counts. http://dx.doi.org/10.1525/auk.2011.10005. 
411 Christie, K. S., S. L. Gilbert, C. L. Brown, M. Hatfield, and L. Hanson (2016). Unmanned aircraft systems in wildlife research: Current and future applications of a transformative technology. Frontiers in Ecology and the Environment 14:241-251.

Darras, K., P. Batáry, B. Furnas, A. Celis-Murillo, S. L. Van Wilgenburg, Y. A. Mulyani, and T. Tscharntke (2018). Comparing the sampling performance of sound recorders versus point counts in bird surveys: A meta-analysis. Journal of Applied Ecology 55:2575-2586.

Ebbert, D. (2019). chisq.posthoc.test: a post hoc analysis for Pearson's chi-squared test for count data. [Online.] Available at https://cran.r-project.org/package=chisq.posthoc.test.

Fu, Y., M. Kinniry, and L. N. Kloepper (2018). The Chirocopter: A UAV for recording sound and video of bats at altitude. Methods in Ecology and Evolution 9:1531-1535.

Gregory, R. D., D. W. Gibbons, and P. F. Donald (2004). Bird census and survey techniques. In

Hioka, Y., M. Kingan, G. Schmid, R. McKay, and K. A. Stol (2019). Design of an unmanned Bird Ecology and Conservation. Oxford University Press, pp. 17-56. and precisely than humans. Methods in Ecology and Evolution.

433 Kloepper, L. N., and M. Kinniry (2018). Recording animal vocalizations from a UAV: Bat 
echolocation during roost re-entry. Scientific Reports 8:8-13.

435

436

437

438

439

440

441

442

443

444

445

446

447

448

449

450

451

452

453

454

455

456

Leitão, P. J., F. Moreira, and P. E. Osborne (2011). Effects of geographical data sampling bias on habitat models of species distributions: a case study with steppe birds in southern Portugal. http://dx.doi.org/10.1080/13658816.2010.531020.

Linchant, J., J. Lisein, J. Semeki, P. Lejeune, and C. Vermeulen (2015). Are unmanned aircraft systems (UASs) the future of wildlife monitoring? A review of accomplishments and challenges. Mammal Review 45:239-252.

Macauley Library. (2014). The Cornell Guide to Bird Sounds: Master Set for North America.

Mennill, D. J., J. M. Burt, K. M. Fristrup, and S. L. Vehrencamp (2006). Accuracy of an acoustic location system for monitoring the position of duetting songbirds in tropical forest. The Journal of the Acoustical Society of America 119:2832-2839.

Miller, D. L., E. Rexstad, L. Thomas, J. L. Laake, and L. Marshall (2019). Distance sampling in R. Journal of Statistical Software.

Mulero-Pázmány, M., S. Jenni-Eiermann, N. Strebel, T. Sattler, J. J. Negro, and Z. Tablado (2017). Unmanned aircraft systems as a new source of disturbance for wildlife: A systematic review. PLOS ONE 12:e0178448.

Newell, F. L., J. Sheehan, P. B. Wood, A. D. Rodewald, D. A. Buehler, P. D. Keyser, J. L. Larkin, T. A. Beachy, M. H. Bakermans, T. J. Boves, A. Evans, et al. (2013). Comparison of point counts and territory mapping for detecting effects of forest management on songbirds. Journal of Field Ornithology 84:270-286.

Nowak, M. M., K. Dziób, and P. Bogawski (2019). Unmanned Aerial Vehicles (UAVs) in environmental biology: A review. European Journal of Ecology 4:56-74.

Pöysä, H., J. Kotilainen, V.-M. Väänänen, and M. Kunnasranta (2018). Estimating production in 
ducks: a comparison between ground surveys and unmanned aircraft surveys. European Journal of Wildlife Research 64:74.

Shankar Raman, T. R. (2003). Assessment of census techniques for interspecific comparisons of tropical rainforest bird densities: a field evaluation in the Western Ghats, India. Ibis 145:921.

Shonfield, J., and E. M. Bayne (2017). Autonomous recording units in avian ecological research: current use and future applications. Avian Conservation and Ecology 12.

Valle, R. G., and F. Scarton (2019). Effectiveness, efficiency, and safety of censusing eurasian oystercatchers haematopus ostralegus by unmanned aircraft. Marine Ornithology 47:81-87.

Wallace, P., R. Martin, and I. White (2018). Keeping pace with technology: drones, disturbance and policy deficiency. Journal of Environmental Planning and Management 61:1271-1288.

Wilson, A. M., J. Barr, and M. Zagorski (2017). The feasibility of counting songbirds using unmanned aerial vehicles. The Auk 134:350-362.

470 Wilson, A. M., K. S. . Boyle, J. L. Gilmore, C. J. Kiefer, and M. F. Walker (2020). SpeciesSpecific Responses of Bird Song Output in the Presence of Drones. Manuscript submitted and E. M. Bayne (2019). Sound level measurements from audio recordings provide objective distance estimates for distance sampling wildlife populations. Remote Sensing in 


\begin{tabular}{|c|c|c|c|c|c|c|}
\hline \multirow[b]{3}{*}{ Species } & \multicolumn{2}{|c|}{ Mapping (territories) } & \multicolumn{3}{|c|}{ Point counts (singing birds) } & \multirow{3}{*}{$\begin{array}{l}\text { song bouts } \\
\text { on airborne } \\
\text { count }\end{array}$} \\
\hline & & & \multicolumn{2}{|c|}{ Terrestrial } & \multirow[t]{2}{*}{ Airborne } & \\
\hline & $\min$. & $\max$. & $1 \mathrm{~min}$ & $5 \mathrm{~min}$ & & \\
\hline Willow Flycatcher & 46 & 50 & 6 & 7 & 10 & 22 \\
\hline House Wren & 23 & 40 & 6 & 10 & 11 & 31 \\
\hline American Robin & 32 & 40 & 4 & 10 & 9 & 52 \\
\hline Field Sparrow & 74 & 85 & 24 & 38 & 43 & 127 \\
\hline Song Sparrow & 52 & 60 & 6 & 15 & 14 & 38 \\
\hline Common Yellowthroat & 44 & 54 & 6 & 11 & 8 & 20 \\
\hline Yellow Warbler & 49 & 57 & 11 & 23 & 8 & 31 \\
\hline Eastern Towhee & 21 & 25 & 13 & 24 & 5 & 8 \\
\hline Northern Cardinal & 41 & 46 & 13 & 25 & 2 & 13 \\
\hline Indigo Bunting & 30 & 34 & 10 & 17 & 10 & 27 \\
\hline all ten species & 412 & 491 & 99 & 180 & 120 & 369 \\
\hline
\end{tabular}

479 Table 1. Estimated number of territories and point count detections (of singing birds) within 100

$480 \mathrm{~m}$ of the point location, for terrestrial counts and airborne counts, 136 ha study area of State

481 Game Lands 249, Pennsylvania, in 2019. 
bioRxiv preprint doi: https://doi.org/10.1101/2020.08.15.252429; this version posted August 16, 2020. The copyright holder for this preprint (which was not certified by peer review) is the author/funder, who has granted bioRxiv a license to display the preprint in perpetuity. It is made available under aCC-BY-ND 4.0 International license.

483

terrestrial airborne

Field Sparrow

78.3

92.4

Song Sparrow

90.1

90.0

all 10 species combined

95.0

90.0

484 Table 2. Estimated detection radii (meters), calculated using R package Rdistance. 


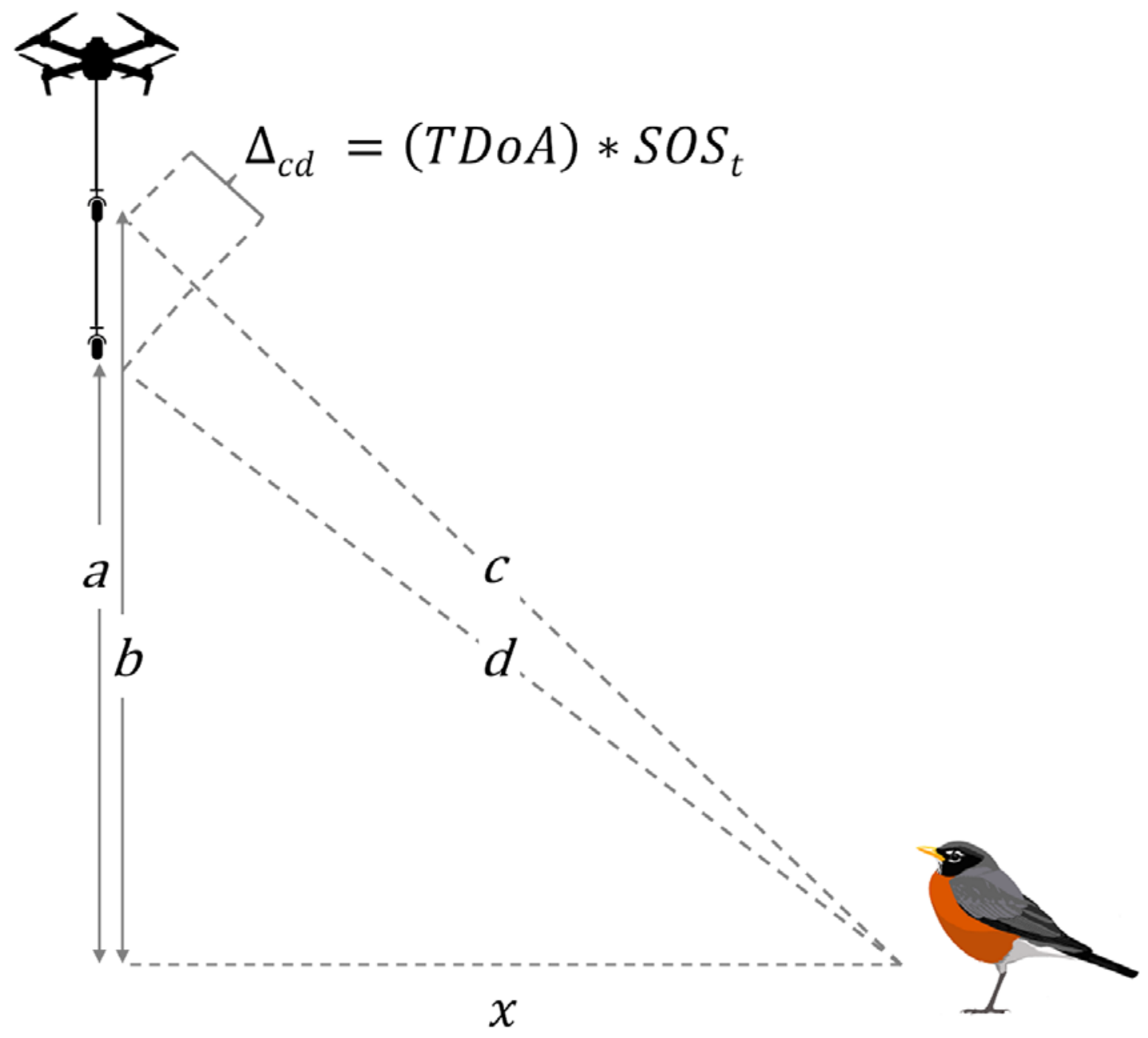

486 Figure 1. Application of the Pythagorean theorem to estimate radial distance from the location

487 under a drone to a bird ( $x$ ), from known heights of two recorders from the ground ( $a$ and $b$ ), and

488 Time Difference of Arrival (TDoA) of a bird vocalization at recorders, based on speed of sound

489 at a given air temperature $\left(S O S_{t}\right) . \Delta_{c d}$ is $c$ - $d$, where $c$ and $d$ are unknown distances, to be

490 estimated using Formula 1. 




492

493 Figure 2. Actual versus estimated radial distances from a location under a drone flown at $55 \mathrm{~m}$,

494 to a broadcast American Robin song at ground level. Error are range bars. 
A

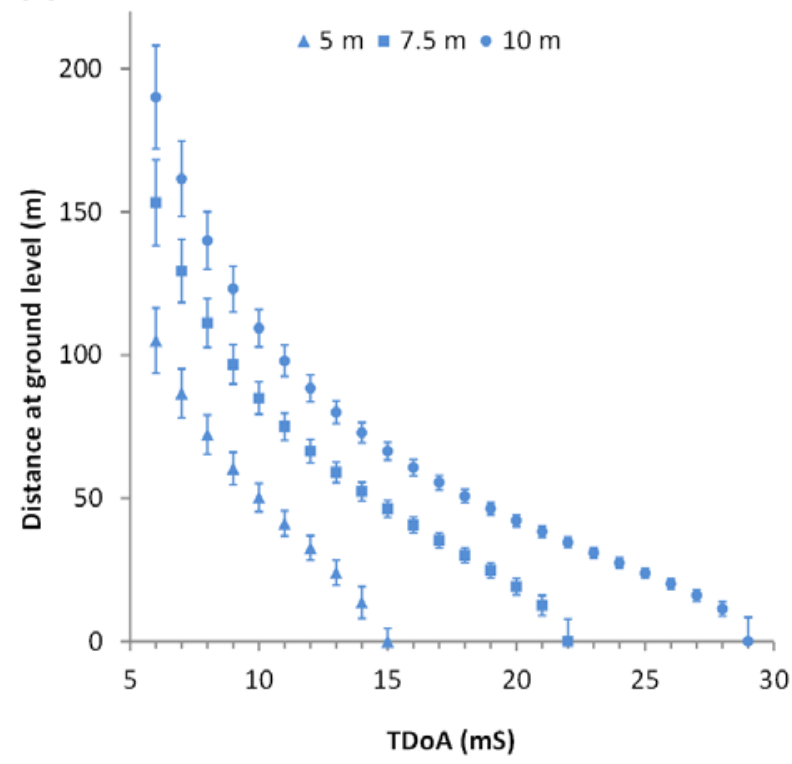

B

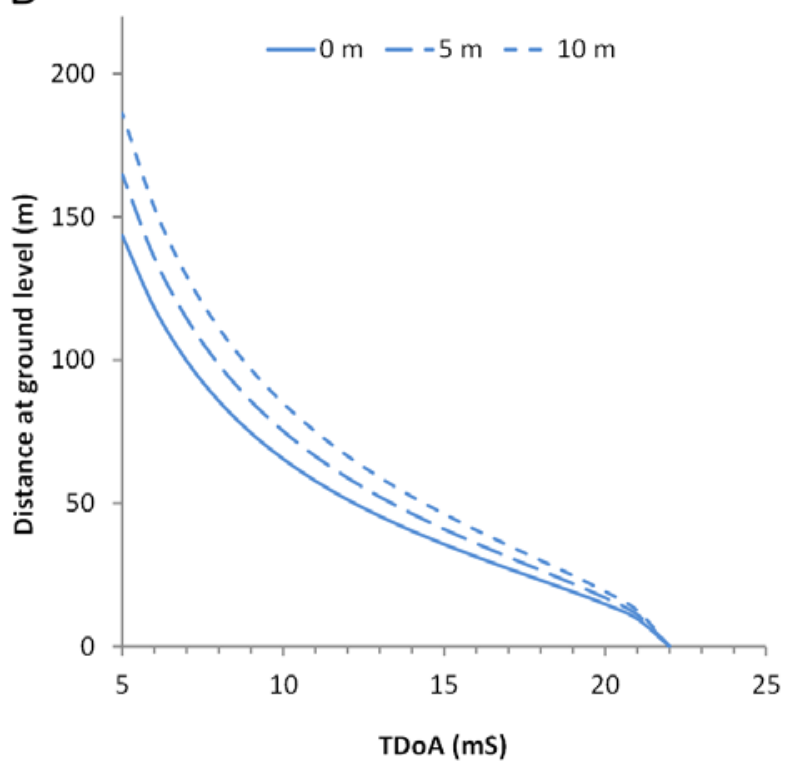

496 Figure 3. A: hypothetical radial distance estimates for measured TDoAs for three different

497 distances between two recorders, assuming an air temperature of $20^{\circ} \mathrm{C}$ and drone altitude of 55

$498 \mathrm{~m}$. B: hypothetical estimated radial distances if it is assumed that a sound source is at three

499 different heights off the ground. 
A

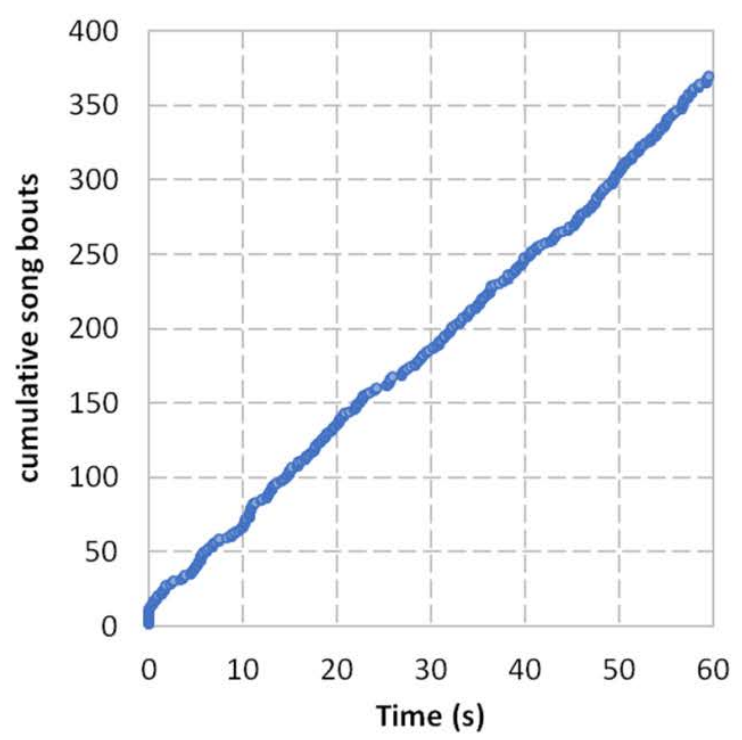

B

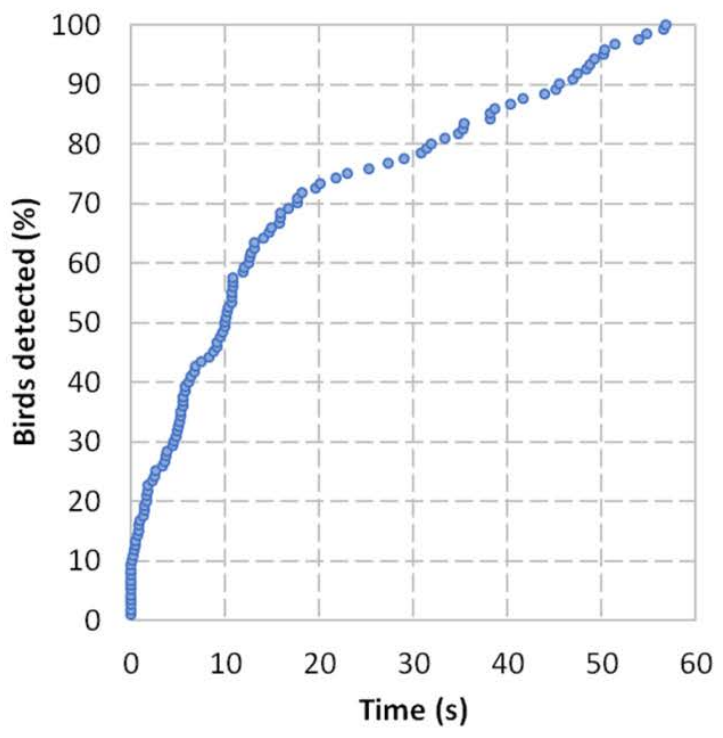

501 Figure 4. A: Cumulative detections of song bouts from airborne counts, across all 34 counts. B:

502 Cumulative percentage of detections of individual birds from airborne counts across all 34

503 counts. 


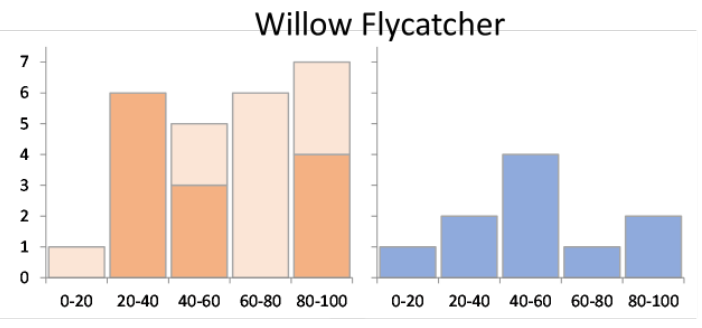

American Robin

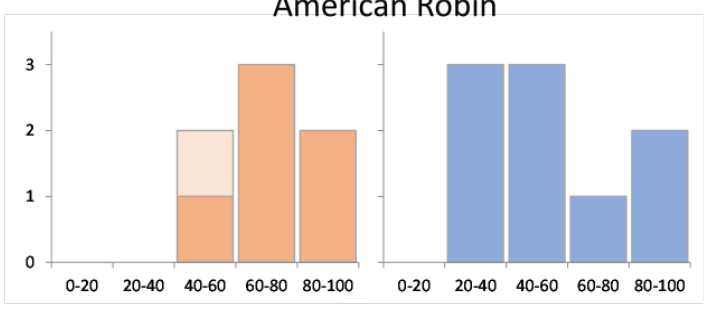

Song Sparrow


all ten species combined

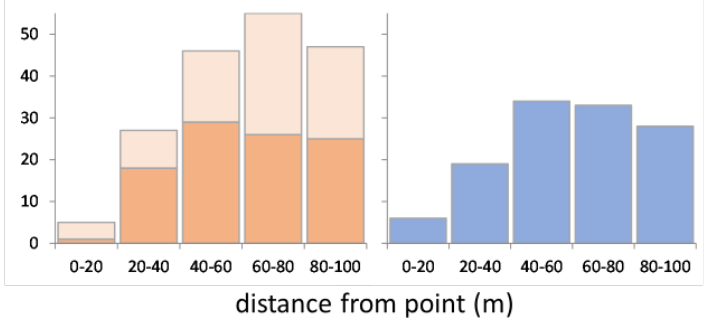

House Wren



Field Sparrow

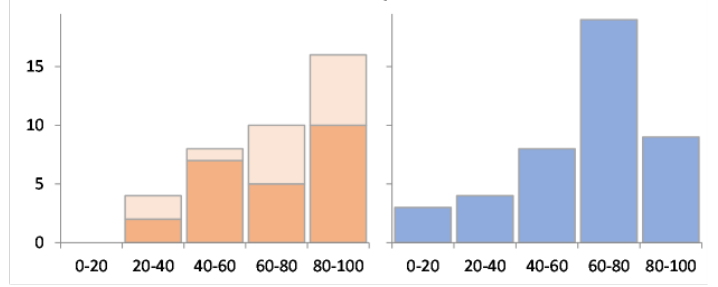

Common Yellowthroat



Eastern Towhee
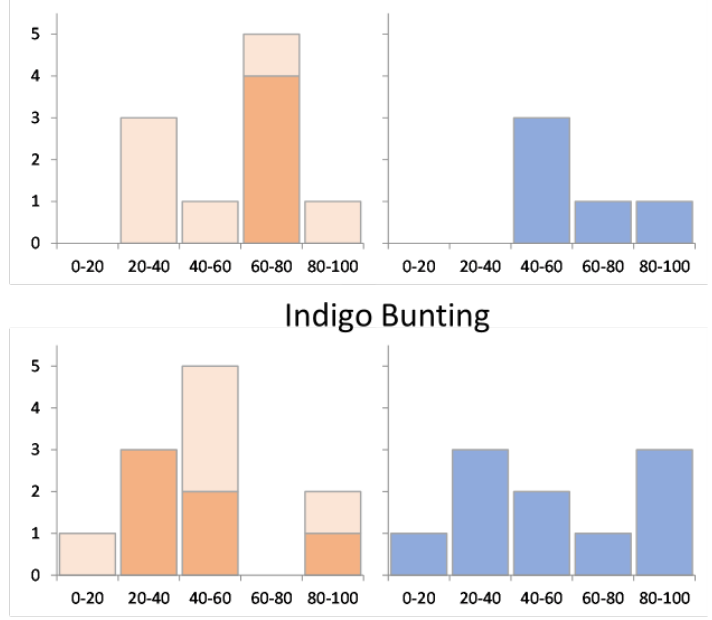

Terrestrial counts

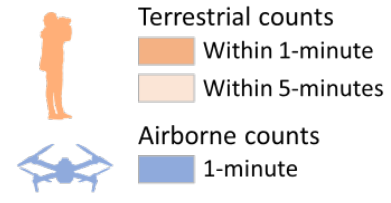

504

Figure 5. Terrestrial and airborne detections of singing birds of 10 species, across 34 point

506 counts, by distance from point count locations. 




508 Figure 6. Comparison of density estimates from mapping (territories) and terrestrial and

509 airborne counts (singing birds) for A: Field Sparrow, B: Song Sparrow, and C: all ten species

510 combined. Error bars for mapping shows the low and high estimates of territories. Error bars for

511 estimates from airborne counts are 95\% CI. CIs were not calculable for density estimates derived 512 from count estimates. 